WEI-LUN LU

Masaryk University, Brno

wllu@phil.muni.cz

\title{
PERSPECTIVIZATION AND CONTEXTUALIZATION IN SEMANTIC ANALYSIS: A PARSIMONIOUS POLYSEMY APPROACH TO IN
}

Keywords: relevance, contextualization, polysemy, perspectivization, Modified Occam's Razor

\begin{abstract}
This paper presents a highly contextualizing approach to the meaning pattern of in. It introduces perspectivization (or vantage point taking) as an important cognitive pragmatic mechanism that accounts for meaning variation of prepositions. In addition, contextualization is included as an important part of the methodology for sense decision. It is hoped that the proposed model can shed light on the connection between cognitive semantics and the cognitive pragmatic principle of relevance.
\end{abstract}

\section{Introduction}

The issue of polysemy is acknowledged as an endemic phenomenon in natural language. As a central pursuit in cognitive linguistics, especially in the field of cognitive semantics, a wealth of polysemy research has emerged since the 80 , with studies on prepositions as one of the mainstreams. These studies centred on semantic approaches to preposition meaning, bypassing the importance of context. The significance of context can be illustrated with the following pair of instances: The patient is in the ambulance / The patient is in pain. A comparison reveals that the meaning of in, with the former as 'physical containment' and the latter as 'state', radically shifts with a replacement of the noun phrase that follows the preposition, i.e. a change in context. Seeing this lack of due attention addressed to context, I will attempt here to deal with the issue of prepositional polysemy from a radical pragmatic stance, 
giving the so-called "context" due attention and will try to bridge the gap of a pragmatic model to the study of polysemy within the cognitive linguistics paradigm.

The present pragmatic approach assumes meaning as contextualization patterns (Taylor 2003) and as an inferential result of perspective taking (Langacker 1987, 1991), with context encompassing immediate linguistic context and shared world knowledge patterns and perspectivization considered a pragmatic strategy that facilitates derivation of meaning in language comprehension. I argue that defining context and perspective taking as such is fundamental to the establishment of a pragmatic model of polysemy.

The preposition investigated is the English in, reflecting the container schema, ${ }^{1}$ which is essential in human perception and cognition (Johnson 1987; Lakoff, Johnson 1999). As previous studies on in are either from a semantic (Lindstromberg 1997; Tyler, Evans 2003; Evans, Tyler 2004) or a pedagogical (Rudzka-Ostyn 2003) point of view, lacking in-depth contextual explanations to the interpretation of prepositions, ${ }^{2}$ a context-oriented pragmatic approach to in is adopted for the present study.

\section{Pragmatic meaning in context}

The context-sensitivity of lexical meaning and the context-dependent fluid nature of utterance interpretation have been reported throughout literature (cf. Cruse 1986; Pustejovsky 1991), but analyses based on a clear and appropriate definition of context are still lacking. Below, I first review the case of safe (Fauconnier, Turner 2002: 25-27) and temperature (Wilson 2003) to illustrate the contextualized nature of interpretation so that an elaboration of the definition of context can be advanced.

\subsection{The context-dependency of interpretation}

Fauconnier and Turner (2002: 25-27) argue that the form of safe prompts "an abstract frame of danger with roles like victims, location, and instrument" and guides us to form a counterfactual blend where participants fit into certain roles based on the situation at hand. The child is safe, for instance, is a typical instance where the child fills in the role of a possible victim, but The beach is safe, in contrast, represents a blending network where the beach is a possible location where harm may happen. Hence, the form safe invokes not only the meaning of 'not harmed' or 'not doing any harm' but an entire set of frame with related participant roles,

See Tyler, Evans' (2003: 180) discussion of in in terms of Lakoff's (1987) container schema of three properties: interio, boundary, and exterior.

2 Although Tyler, Evans (2003) and Evans, Tyler (2004) emphasize the importance of context, their approach simply stresses certain part of the notion: "word meaning is context-sensitive drawing upon encyclopedic knowledge as well as inferencing strategies which relate to different aspects of conceptual structure, organization and packaging" (Evans, Tyler 2004: 159). The present research moreover incorporates the immediate textual context and the physical context into the scope of study. Apart from that, my radical pragmatic approach also results in a decisively different role that context plays in sense establishment. 
and its interaction with its immediate linguistic context such as child or beach also involves the knowledge base of a child as being vulnerable and that of a beach as a potentially dangerous place.

Wilson (2003) discusses that the temperature as in I have a temperature triggers a scalar term, with different points on the scale carrying different implications under contextual assumptions. Heard in a scenario of invitation, the utterance of temperature will denote a temperature high enough to prevent the addresser from accepting the invitation. The contextual assumptions will pertain to our encyclopedic knowledge of temperatures and the possible consequences of accepting an invitation under a high temperature.

In sum, both studies concern the interpretation of a lexical item given certain contextual assumptions. However, note that the notion of contextual assumption is addressed under a covering umbrella without a delineation of its detailed components, thus before I proceed onto our case of the contextualization of $i n$, a clearer definition of context is necessary.

\subsection{Defining context}

Seeing the vagueness of context, Croft and Cruse (2004: 102-103) generalize a fourfold classification of the term, inclusive of linguistic context, physical context, social context, and stored knowledge. Accordingly, for the cases of safe and temperature, the contextual assumptions will refer first to the linguistic elements that occur around the target lexeme such as child or beach, to the stored world knowledge of a beach, child, or the experience of running a high temperature, and also to the situational context where the utterances are heard.

Among the four types of contexts, linguistic context, physical context, and stored knowledge concern the purpose of the present study the most, especially linguistic context and stored knowledge. ${ }^{3}$ Linguistic context, according to Croft and Cruse, includes "previous discourse", "immediate linguistic environment", and "types of discourse". For the sake of a study on prepositions, the "immediate linguistic environment" calls for more emphasis because a preposition indicates the relationship between two noun phrases, and it is the noun phrases that exert most influence on the interpretation of the preposition. Hence, for the purpose of the present paper, two major types of context will be specifically addressed: The type of linguistic context that pertains to a preposition study will be its immediate linguistic environment, viz. the lexical expressions that occur in the surroundings of the target preposition. ${ }^{4}$ On the other hand, the stored knowledge refers to "a vast store of remembered

3 This is not to entirely exclude the social factor from the understanding of prepositions. The point here is simply that for the several meanings scrutinized in this study, the other three types of context are of higher significance. Another point to make here is: it is also likely to incorporate social factors as a part of stored background knowledge, so the label of social context may not be necessary.

4 For the purpose of this study, linguistic context is used in a similar sense to co-text or textual prompt (Lu 2008). 
experiences and knowledge" against which utterances are processed (Croft, Cruse 2004: 103). The stored knowledge will thus involve related frames that structure our understanding of a concept, and experiences of our interaction with the environment.

\subsection{The role of context in previous studies on in}

In and its cognates have been widely studied throughout literature (Miller, JohnsonLaird 1976; Hawkins 1988; Herskovits 1986, 1988; Vandeloise 1991, 1994; Dirven 1993; Hottenroth 1993; Lindstromberg 1997; Rudzka-Ostyn 2003) but due to the highly semantics-based nature of the above studies, the issue of contextualization and its influence on prepositional meaning has not received due attention, with the only exception of Tyler, Evans (2003) and Evans, Tyler (2004), which propose a critical notion of proto-scene and its relation with embodied meaning.

Tyler, Evans (2003) and Evans, Tyler (2004) propose a schematic spatial representation termed the proto-scene from which all the other senses emerge. Two important elements constitute the proto-scene: an abstract spatial representation of a Trajector (TR) and a Landmark (LM), ${ }^{5}$ and the functional element, which "reflects the interactive relationship between the TR and LM, and... the meaningful consequences to us" (Tyler, Evans 2003: 230). Following the authors, I consider the protoscene the primary sense from which the other meanings derive. However, a different theoretical concern orients the present study towards a contextualized approach to polysemy in that their semantics-based approach renders at least 12 senses, most of which will be considered context-dependent interpretations rather than separate senses given clearly defined pragmatic criteria. ${ }^{6}$

Specifically, the functional element in Tyler and Evans' Principled Polysemy will be put in a different theoretical position in the present one provided that our definition of context includes "linguistic context" and "stored knowledge". The functional element, according to them, refers not only to the interaction between the TR and the LM, but also to human embodied experience related to the interaction between the TR and the LM. The functional element, in other words, represents the concept of containment in our case of $i n$, and how human beings conceptualize and make use of the idea of containment. The role of such embodied experiences, to the contrary, will not be integrated in the sense of preposition per se, but is instead put under the umbrella of context in that embodied experiences are cumulated patterns of human knowledge of their interaction with the world. Hence for the contextoriented nature of the present study, a reassignment of the embodied experience as

A TR and a LM, according to Langacker (1987), stand for the primary and secondary figure in conceptualization respectively. A landmark serves as ground for the trajector (Croft, Cruse 2004: 58). In the instance of The cat jumped over the wall (from Tyler, Evans 2003), the wall functions as the landmark with which the relative location of the cat is marked.

6 In this study, I reserve the label interpretation for ostensively communicated meanings that extend from the processes of pragmatic inferencing from its literal/central lexical meaning given appropriate contextual prompts, as opposed to senses that do not vary with context and are entrenched (Langacker 1987) as a separate lexical entry. 
a type of context is necessary, and such reorganization will prove to bear a significant consequence to the establishment of a cognitive-pragmatic model of polysemy and to the relative position of pragmatics within cognitive linguistics.

In sum, the present radical context-driven model of polysemy approach the meaning of prepositions from a radically different perspective from previous semanticsbased ones, with the notion of context clearly redefined and receiving relatively more attention.

\subsection{Perspectization: Vantage point shift and flexibility of meaning}

In this paper, I consider perspectivization, or vantage point taking, another force that operates on meaning in context. Langacker $(1987,1991)$ proposes that the notions of orientation and vantage point are subsumed under the umbrella of perspective, and a particular type of viewing arrangement constitutes an intrinsic aspect of a linguistic form's semantic value, which is often the case for deictic expressions such as left, right, yesterday and tomorrow. For instance, uttered in the context where the speaker sits face to face with Raisa and Nancy, the sentence Raisa was seated on Nancy's right (Langacker 1991: 315) can bear two entirely different interpretations: with Nancy's point of view taken, the sentence represents a spatial configuration as in Figure 1a; with the speaker's vantage point taken, the sentence can denote an opposite spatial relation shown in Figure 1b, where the solid circles stand for the respective vantage points:
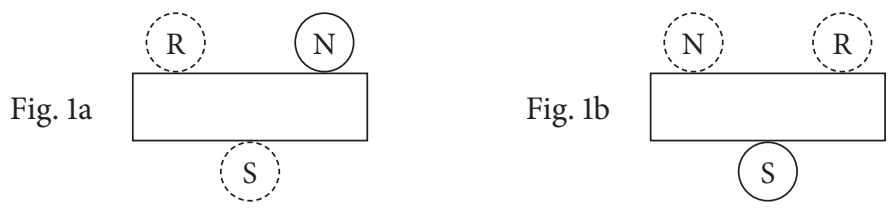

Figure la and 1b. Different spatial representations based on different viewpoints

Aside from the role of context in the model of polysemy, another fundamental disparity between the present study and Tyler, Evans (2003) and Evans, Tyler (2004) is the status of vantage point choice within our respective models of polysemy. In their semantics-based model of prepositional polysemy, the cognitive mechanism of vantage point shift discriminates 'perceptually accessible' and 'disappearance' as two separate senses. Perspectivization is in other words a criterion that tells senses apart in their studies. On the contrary, I consider perspective taking as a cognitivepragmatic operation, which simply modulates interpretations in context rather than draws a line between senses.

\subsection{Methods and criterion for sense establishment}

The study follows Sperber and Wilson $(1986,2002)$ and Carston (2002) in treating pragmatics as an information processing system that works with a subset of assumptions of the interpreter. Meaning is in other words an inferential product of 
the integration of basic lexical properties and surrounding contextual information, tuned up via cognitive-pragmatic operations. Preposition-wise, if a meaning of a preposition can be accounted for by the interaction between its basic property and relevant contextual inputs, then it should be conservatively categorized as a contextdependent interpretation instead of as a distinct sense. Hence Grice's (1978) Modified Occam's Razor is incorporated as the guideline for sense establishment: "Senses are not to be multiplied beyond necessity". The criterion discriminates situated interpretations from senses with extreme caution in terms of the degree of "necessity" and will thus bear a significant analytical result to the proposed model of polysemy. The in as in What are you in for? [asked in a hospital = 'what's wrong with you?'], for example, is analyzed as 'the in situ sense,' by Tyler and Evans (2003: 187). But such a "sense" is in essence a result of the intervention of the encyclopedic knowledge associated with the pragmatically retrievable but omitted noun hospital. Such reading of $i n$ is in other words to be attributed to the interaction of the basic property of the preposition and the contextual cues, i.e. the physical context in which the utterance is used, and the knowledge triggered by the noun, i.e. knowledge of a hospital as a place of a specific attendant purpose. Based on the criterion, the meaning 'in situ' will not have to be sanctioned as a distinct sense but will be seen as an interpretation that is dependent on its immediate textual prompt.

It must in addition be noted that the analysis in the present study does not intend to cover every single occurrence of in. ${ }^{7}$ Previous studies propose a diverse range of meanings and different labels for those meanings. Without factoring context into sense establishment, those semantics-based studies nevertheless tend to suffer the criticism of being overly fine-grained. ${ }^{8}$ It would hence take up too much space for me to argue for/against the status of every single meaning as a distinct sense one by one, so as the first step, meanings that are mentioned by more than one study are dealt with to establish a preliminary model of the polysemy of in, after which the rest of the meaning labels are checked against this model to attest their status. I believe that such practice will suffice to illustrate how my heavily contextualizing approach differs from the previous semantics-based ones.

\section{Meaning patterns of in and contextualization}

I list four meanings that have been identified as senses of in in the previous studies for their relative significance: 'physical containment', 'state/situation', 'temporal containment', and 'way/means'. These meanings, which reflect the effect of contextual modulation (Cruse 1986) of the immediate surrounding linguistic environments on in, will be discussed in order below from 3.1. to 3.4.

Only senses that are mentioned by more than one study are included in the scope of our discussion for the limit of length.

8 With the term polysemy's fallacy, Sandra (1998) airs complaint regarding the over-exaggeration of polysemy and the shortcoming of sense proliferation in many cognitive linguists' analyses. 


\subsection{Primary sense: 'physical containment ${ }^{\prime 9}$}

'Physical containment' is a meaning that is mentioned in all the four studies, and its status as the prototypical meaning is agreed upon here. Following Tyler and Evans' idea of the proto-scene, I also recruit such basic physical meaning as the primary sense from which all the other meanings derive in context. The proto-scene is diagrammed as a TR located within a LM, represented with the circle and the box in Figure 2 respectively:

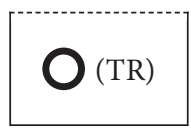

(LM)

Figure 2. Proto-scene for in (after Tyler, Evans 2003: 184)

Tyler and Evans and Lindstromberg also place considerable emphasis on the spatialconfigurational relation between the TR and the LM, discussing what counts as a physical containment sense of $i n$. Consider the typical cases of 'physical containment' below:

(1) The cow munched grass in the field. (Tyler, Evans 2003: 184)

(2) We got in the car. (Lindstromberg 1997: 29)

In the above instances, the cow and we are TRs of the conceptualization, while the field and the car are regarded as the LMs. The TRs are notionally thought of as being "contained" in the LMs via the linguistic use of in.

\section{2. 'State/situation'}

A major extension from the proto-scene is the meaning of 'state/situation'. Rudzka-Ostyn and Lindstromberg both distinguish between the sense of emotion states and the sense of situations, but the meaning can be covered under the label of the State Sense proposed in Tyler, Evans (2003: 187-189). The following examples are representative of the meaning of 'state/situation':

(3) She is in prison. (Tyler, Evans 2003: 188)

(4) She's in a deep depression. (Lindstromberg 1997: 75)

As has been mentioned by Tyler and Evans, the functional element of in is containment, which poses "difficulty in leaving" (Tyler, Evans 2003: 188). The linguistic context in the first instance is prison. A prison, based on human encyclopedic knowledge, is a place of no easy escape, and once getting in there, one usually stays for a long time.

9 I follow Evans and Tyler's (2004) terminology of "primary sense" here. However, it should be noted that the labels "primary sense", "sanctioning sense" and "proto-scene" seem interchangeable in Evans, Tyler (2004). 
Therefore such context of stored knowledge reveals that being in a prison represents lengthy confinement, which is triggered by the textual prompt of prison. A deep depression is also something difficult to rid of based on our knowledge. A state of deep depression thus corresponds to the functional element of containment given the use of in and its interaction with its linguistic context. Accordingly, the undergoer of the state/situation is viewed as the TR and the state/situation as the LM, with the interpretation of "TR being in the state of contained and thus having difficulty leaving" prompted by the co-text $a$ deep depression and the related stored knowledge. Therefore, the meaning of 'state/situation' in the above examples is derived from the interaction of the functional element of containment in the proto-scene, the immediate linguistic context, and the world knowledge associated with the linguistic context. With contextual assistance, 'state/situation' can hence be considered a pragmatic extension from the proto-scene by the principle of parsimony.

\section{3. 'Temporal containment'}

The meaning of 'time' is listed as a separate sense by Lindstromberg and Rudzka-Ostyn. Typical examples include:

(5) I will go there in spring. (Rudzka-Ostyn 2003: 52)

(6) In the evening we sat around and drank beer. (Lindstromberg 1997: 77)

In the above examples, the co-text that occurs with in is spring and evening. Related to the lexical items is our knowledge of these temporal units as units long enough to be conceptualized as a container, as is argued by Lindstromberg (1997: 75). The textual prompts and the stored knowledge combine with the functional element of containment, which invokes the conceptual product of a metaphor TIME IS SPACE (Lakoff, Johnson 1980, 1999; Grady 1997). Such conceptual metaphor can be considered a type of shared assumption as context, in that they are also an emergent notional pattern by which human beings grasp temporal concepts. Via such collaboration of textual prompt, encyclopedic knowledge and proto-scene, the events at issue are viewed as TRs and the temporal units as LMs, with an interpretation of "the temporally more compact TRs being included in the longer LMs". Hence 'temporal containment' is also regarded as a context-dependent use extended from the functional element of containment, with the event coded considered the TR and the temporal frame the LM that metaphorically 'contains' the event, as a result of the interaction between the proto-scene, the surrounding linguistic context, and encyclopedic knowledge.

\section{4. 'Way/means'}

The meaning of 'way/means' is proposed by Tyler and Evans, which covers Lindstromberg's (1997) 'linguistic expressions are containers' ${ }^{10}$ in that linguistic

10 This seems to correspond to Reddy's (1979) conduit metaphor. 
expressions can also be viewed as a means of communication. The instances below are typical cases:

(7) In other words, ... (Lindstromberg1997: 75)

(8) She wrote in ink. (Tyler, Evans 2003: 190)

The derivation of the meaning is opaque, but Tyler and Evans proposes a possible motivation for the meaning as an extension from 'activity' due to the tight correlation between the means of accomplishing an activity and the activity itself. However, by observing this set of examples in a way / in this way / in many ways, I have reached an alternative account for its derivation.

A common characteristic in this set of instances is the collocation of way with in in the constructions. The textual prompt invokes an event-structure metaphor MEANS ARE PATHS (Lakoff, Johnson 1999: 179), which serves as the shared knowledge base on which we understand the utterance. The use of in is to create a construal of the path/way as a conduit-like container that limits and directs the means by which the event coded is carried out. Thus a brief look at this set of examples explains the possible origin of 'way/means' of in as basing itself on the coordination of co-text and the functional element of containment, and may lead us to a conclusion of 'way/ means' as a mere derived use in context. It should nevertheless be noted that such meaning also occurs in constructions without a collocation of way with in, such as examples (7) and (8), where the above textual cues are no longer available for prompt of the conceptual level operation. Therefore, according to the proposed methodology, 'way/means' cannot be a contextual implicature but can only be considered a discrete sense, as the meaning occurs without contextual contribution in most cases. In other words, the original context-situated use can be argued to have become a well-entrenched sense of the preposition in and can happen in various contexts.

\section{More than context: Vantage point shift}

I have up to this point presented four meanings introduced by more than one of the previous studies: 'physical containment', 'state/situation', 'temporal containment', and 'way/means', with 'physical containment' as the prototypical sense from which the other meanings derive. 'State/situation' and 'temporal containment' are analyzed as context-sensitive uses only since the meanings are pragmatic products of the interaction of the functional element of containment and immediate textual cues rather than intrinsic semantics of the preposition itself. These interpretations are, in other words, a result of contextual modulation arising out of the interaction between the proto-scene of in and its relevant contextual information. On the other hand, I suggest 'way/means' be listed as a distinct sense, given that no relevant co-text is available in most of its occurrences albeit its path of meaning extension is accountable.

In this section, I discuss perspective taking (Langacker 1987), another cognitivepragmatic mechanism that modulates the meaning of a preposition in language 
comprehension: meanings to be discussed that involve the such cognitive-pragmatic mechanism are 'appearance' and the phrasal verb construction fade in as a special case of 'appearance' in 4.1., and the meaning 'disappearance' as opposed to 'appearance' in 4.2 .

\section{1. 'Appearance'}

'Appearance' is a meaning that covers two distinct senses 'arrival' and 'perceptually accessible' reported in Tyler, Evans (2003) and Evans, Tyler (2004). The derivation of 'arrival' and 'perceptually accessible' and their relation with respect to perspectivization will be addressed in 4.1.1. and 4.1.2. In 4.1.3., I argue that, encompassing the above two meanings that concerns perspective taking, in 'appearance' certainly involves the conceptualizer's perspective located within the container.

\subsection{1. 'Arrival'}

'Arrival' is a meaning that is discussed as a distinct sense by Tyler and Evans. The following examples are taken from their discussion (Tyler, Evans 2003: 194):

(9) The train is finally in.

(10) She clocked/punched in at work.

A common feature of this meaning is the involvement of physical motion of the TR into an unspecified LM. The meaning of physical motion is also derivable from the immediate contexts of a train coming to the station and an employee going into her office, and an example without physical motion such as Sorry. I'm afraid John is not in now reveals the context-dependent nature of 'arrival'." Another proof comes from the mystery of the recovery of the unspecified LM. The reason why language users are able to recover the unspecified LM is also attributed to the shared world knowledge of train as being tightly associated with a station or the platform, and an employee punching in at work as closely related to her office. Such patterns of assumption help us retrieve the unexpressed LMs.

But the TR moving into the LM does not necessarily mean 'arrival' because the concept of 'arrival' requires, apart from physical motion, a reference point, viz. the location at which the TR comes to a full stop. If the reference point coincides with the position of the TR, i.e. within the container, the interpretation of 'the TR moving into the state of being contained' readily becomes 'the TR moving to the physical location of the observer'. Such perspective taking thus turns the meaning of 'physical containment' to 'arrival'. Hence the derivation of the situated interpretation of in as 'arrival' requires the following conceptual components, including a linguistically manifested TR, its notionally traceable LM triggered by shared

11 As the example has shown, the retrieval of LM information may also depend upon the physical context as well instead of simply on the linguistic context and stored knowledge. 
knowledge, a perspective shift from the outside to the inside of the container, and the human inferential capacity that may arrive at the interpretation of 'arrival'.

\subsection{2. 'Perceptually accessible'}

Another meaning tightly associated with 'appearance' is 'perceptual accessibility'. It is reported also by Tyler and Evans (2003: 191) as a consequence of "the experiencer and vantage point being located within a bounded LM", which is "a significantly different occurrence from that which takes place when the experiencer is located outside the bounded LM". The following are representative instances:

(11) I have it in view.

(12) I have him in sight.

In (11) and (12), the textual prompts are view and sight, which triggers the encyclopedic knowledge of the visual field and our embodied experience of the visual field usually considered a container. Moreover, the vantage point is naturally in the LM since someone's viewpoint must co-occur with his visual attention as a result of human bodily limit. That is, the vantage point shift is, more or less indirectly, triggered by the co-text, due to the related knowledge of the visual field, and the embodied experience of the visual field as a container and the fact that one's point of view necessarily resides in the "container". With the entity observed construed as the TR and the visual field as the LM, the meaning of 'perceptually accessible' is actually an interpretation of "the TR included in the container of the LM, the visual field" with a vantage point that resides in the container. Hence the interpretation of in as perceptually accessible is a natural inferential result of the combination of the functional element of containment of the proto-scene, the linguistic context, perspective taking, and shared embodied patterns, so should be analyzed as a pragmatic use in context.

\subsection{3. 'Appearance' and vantage point shift}

So far, I have established 'arrival' and 'perceptually accessible' as interpretations as a result of the interaction of the proto-scene, the following two elements of context: the immediate linguistic cues surrounding in, and stored knowledge patterns associated with such cues, and, more importantly, a conceptual vantage point located within the container. It has furthermore been argued that the derivation of 'arrival' is based on the contextual elements denoting physical motion, and the visual container as triggered by the textual prompts sight and view.

Here, I further propose an abstracted meaning of 'appearance' that is generalized from the above two, since 'arrival' can be understood as 'appearance in the physical container (or the unspecified but recoverable LM)' and 'perceptual accessibility' as 'appearance in the observer's visual container'. 'Appearance' will, following the discussions of the above two meanings, thus be considered a pragmatic interpretation also involving a vantage point within the container. Figure 3 demonstrates such perspective taking, where the eye-like icon represents the vantage point inside the container. 


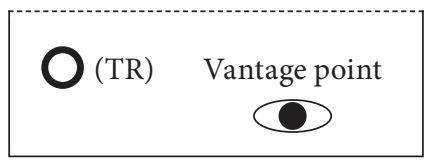

$(\mathrm{LM})$

Figure 3. 'Appearance' and the vantage point inside the container

\subsubsection{Wag the dog: The radical case of fade in}

It has been the major claim of the discussion up to this point that in receives various contextual inputs and can develop context-sensitive interpretations from its proto-scene. However, in this subsection I present a different case - the phrasal verb fade in, where in contributes the meaning of 'appearance' as well. I argue that this particular case is most representative of the interdependent nature of contextual modulation in that the preposition has, as a part of the immediate linguistic context for a verb, modulated the verbal meaning in return. The following are typical instances of fade in:

(13) This means the caption will fade in over 1.5 seconds when it first appears on the screen. ${ }^{12}$

(14) In order to see your panels, you hit a function key and they fade in over the top. ${ }^{13}$

In the examples the meaning of fade in is 'to appear gradually'. ${ }^{14}$ However, the meaning of 'gradual appearance' is not reflected in the semantics of fade, which includes: 'become less clearly visible or distinguishable; disappear gradually or seemingly', 'lose freshness, vigor, or vitality', 'disappear gradually' and 'become feeble'. ${ }^{15}$ But none of the above meanings matches what we see in fade in, which seems to have an opposite meaning to the semantics of fade. In other words, the meaning of 'decrease' or 'disappear' in fade is, so to speak, cancelled, or even reversed by the intervention of in given 'appearance' as one of the meanings in use of the preposition, as has been mentioned in 6.1. Therefore, with the extreme case of fade in, I have demonstrated the power of context as not merely capable of adjusting the meaning of a preposition, but also capable of inverting the meaning of the verb in a verb particle construction.

\subsection{Disappearance}

'Disappearance' is a distinct sense discussed also in Tyler, Evans (2003) and Evans, Tyler (2004). The explanation based on the functional element of the proto-scene is that

12 Authentic instance taken from the internet [www.adobe.com/devnet/captivate/articles/ demo_simulation_o6.html].

13 Authentic instance taken from the internet [desplesdadotcom.nfshost.com/? $\mathrm{p}=75$ ].

14 Definition from the Online American Heritage Dictionary, Fourth Edition [www.bartleby.com/ 61/92/Fooo920o.html].

15 From WordNet Search 2.1 [search date: 2006/6/21]. 
"the boundary of the LM often obstructs the observer's view of the interior and hence the contents". This is evidenced by the instances given below (Tyler, Evans 2003: 195):

(15) Millie rubbed in the lotion.

(16) The sun is in.

The TRs here are lotion and the sun with respect to in, while the LMs are, quite similar to those in the meaning of 'arrival', unspecified but retrievable. The retrieval of the LMs is analogously dependent on the encyclopedic knowledge associated with lotion and the sun: it is almost always the skin on which the lotion is applied and which absorbs the lotion and the mountains that obstruct the sun at sunset. The linguistic cues of the TRs, along with the shared assumptions, thus help in the recovery of the LMs. Aside from that, such meaning extension from the functional element of containment to 'disappearance' requires a vantage point located outside the container since the boundary of the LM, as is argued by Tyler and Evans, prevents the TR from being visible, as is illustrated in Figure 4. Hence the understanding of in as 'disappearance' in these instances is a derivation from the functional element of containment given the textual cues and the knowledge associated with the textual cues, and more importantly a vantage point outside the container from which the vision is blocked by the boundary of the container. 'Disappearance' is therefore a pragmatic interpretation arising from an integration of the functional element of containment of the proto-scene, the immediate linguistic context, the knowledge triggered by the textual prompt, and a specific location of the vantage point outside the container.

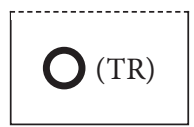

Vantage point

(LM)

Figure 4. 'Disappearance' and the vantage point outside the container

\section{Towards a parsimonious representation of polysemy}

Our discussions so far have implicated the beauty of an extreme pragmatics-based model of prepositional understanding. Below, I address three main implications born out of the discussion above: contextualization and perspectivization, and how cognitive pragmatics, specifically the relevance-theoretic approach sheds light on theorizing polysemy.

\subsection{Contextual implication on polysemy}

Negligence of context has put previous semantics-oriented models of polysemy in the doubt of sense proliferation. My pragmatic approach in contrast well captures 
the essence of contextual influence on prepositional meaning by clearly redefining context as incorporating immediate linguistic environment, physical environment and the interpreter's existing background assumptions. I further adopt a rigorous criterion of sense establishment based on the principle of parsimony, shaving off unnecessary "senses" if contextual factors can be found in effect in the derivation of the meaning discussed.

The meaning of 'temporal containment' and 'state/situation', for instance, has been given a status of interpretation given their context-dependency, while 'way/ means' is entitled to establish as a sense since a combination of primary meaning and context alone does not sanction its derivation from the meaning of 'physical containment'. Hence by such standard, the present contextualizing approach has dramatically reduced the number of senses established for in from Lindstromberg's (1997) eleven, Rudzka-Ostyn's (2003) six, or Tyler, Evans' (2003) at least twenty-three with six clusters to only two, so is highly economical in the sense that the burden of sense proliferation can be efficiently alleviated.

\subsection{Pragmatic consideration for the perspectivized interpretations}

In addition, in the present account, the problem of sense proliferation is also mitigated with an eye on the issue of perspective shift. Section 4 has introduced a few meanings involving the pragmatic operation of perspective choice: 'arrival', 'perceptually accessible', 'appearance' as a generalization from the above two, and 'disappearance'. Our discussion also reveals that the polar meanings carried by in are essentially an inferential consequence of different perspective alignments, with 'appearance' involving a vantage point inside the container and 'disappearance' one outside the container. ${ }^{16}$

The meanings of 'appearance' and 'disappearance' look semantically contradictory, but are both analyzed as context-sensitive extensions from the primary meaning of 'physical containment' in the present model. The cognitive pragmatic approach to prepositional meaning also captures the underlying reason behind such sharp contrast: shift in the vantage point. For 'disappearance', the vantage point is located outside the LM, so the TR is obscured from the observer once it enters the LM. To the contrary, for 'appearance', the vantage point coincides with the internal of the LM, so when the TR moves into the LM, it becomes visually present to the conceptualizer. The cause of the opposite interpretations of in as visually present or obscure boils down to their fundamental difference in perspective taking and the interaction between the vantage point and the relevant container in question. The peculiar phenomenon of meaning opposition in one lexical item thus does not

${ }^{16}$ A further comparison of this pair can attest the context-dependent nature of 'appearance' and 'disappearance': The train is in / The sun is in. With almost identical contexts except for the preceding noun phrases, the former in means 'appearance' and the latter 'disappearance'. That is to say, the respective meanings rely on their immediate linguistic context train and sun. It is thus safe to claim the situatedness of the two uses. 
come from the semantics of the preposition per se but instead can be attributed to the pragmatic operation of perspectivization.

A definition of perspective shift as a pragmatic mechanism bears significant analytical consequences: the distinction between 'appearance' and 'disappearance' becomes pragmatically derived, which renders them only pragmatic interpretations instead of discrete senses given the criterion of Modified Occam's Razor. Figure 5 thus summarizes the analytical result of the meaning pattern of $i n$ from the present context-oriented perspective:

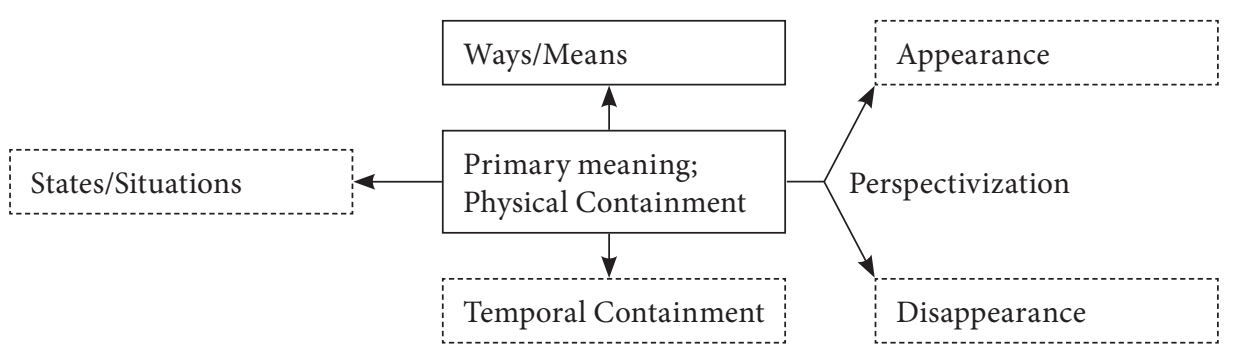

Figure 5. Proposed pragmatics-based meaning network of in

\subsection{How the principle of relevance sheds light on prepositional polysemy}

I moreover argue that the proposed model of prepositional polysemy is highly economical. But how does one assess such theorizing of polysemy model from a cognitive-pragmatic perspective? The first and foremost question to face is: the idea of context, or interpreter's background assumptions, is infinite and immense, which may include every piece of possible knowledge possessed by human beings. Therefore, a question that naturally follows is: how much should one include in the context in processing a lexical item? Put polysemy-wise, where does a meaning stop to be an interpretation and start being a sense?

One is constantly and truly faced with such an analytical dilemma as a linguist, and debates concerning how polysemous a preposition should be are evidenced by the incongruency among studies - the variation among the studies on in is quite telling. One can certainly propose an extreme fine-grained approach to prepositional meanings like what Lakoff (1987) does with over 40 senses advanced for over. One can by contrast empower the pragmatic or inferential capacity to the extreme, which is exemplified by Ruhl's (1989) monosemy approach. It seems that the varying weight of pragmatics in lexical semantic analysis essentially results in the surface divergence of theoretical orientation among different polysemy models.

Given such theoretical distinction, I further argue that such fundamental difference in degree of reliance on the human inferential capacity can be nicely captured by the principle of relevance advanced by Sperber and Wilson (1986). The authors argue that human communication and cognition is geared in search of maximal contextual effects and minimal processing effort: a hypothetical purely semantics-based approach to prepositional meaning would include least contextual information into the 
decoding process, yet its processing effort would also be minimal; an extreme pragmatic approach would incorporate the most knowledge possible as its context with the highest degree of contextual effects as a result, yet more processing effort would also be required as a tradeoff. Either approach enjoys its own merit yet bears certain unsatisfactory disadvantage in terms of contextual effects and processing effort.

To the division, a valid question can be asked: is an ideal cut-off point possible where the greatest relevance can be achieved? The answer remains unfortunately vague for the time being, yet a contrast between two instances analyzed in the present study allows for a clearer picture. Consider first the comparison of 'appearance' and 'disappearance' discussed in an earlier section with The train/sun is in as illustrations. In this pair, the interpretation of in varies with the noun phrase train/sun in its immediate linguistic context, and the cognitive mechanism of vantage point shift is in effect. It is obvious that such replacement of nearby noun phrases triggers a high contextual effect by inducing the user to tap into the world knowledge associated with respective noun phrases and thus achieves considerable relevance. Therefore, an attribution of the antonymous polysemy to the inferential capacity is significant in that such practice highlights the role played by context and relevance in how humans use language.

On the other hand, the 'means/way' sense may serve as a contrast to the above instance: as has been argued, the derivation of the meaning requires an event-structure metaphor MEANS ARE PATHS. I have also discussed that the textual prompts of in 'means/way' rarely invoke such event-structure metaphor, so that it makes better sense to treat the above meaning as a context-independent sense. One could otherwise consider MEANS ARE PATHS to be a part of encyclopedic knowledge that resided in our long-term memory, which would give rise to an analytical result of 'means/ way' as a context-dependent meaning for the inclusion of the conceptual metaphor as a part of context in a broad sense. Nevertheless, such extreme pragmatic treatment would raise the processing effort by expanding the scope of existing background assumptions without limit to try to incorporate every piece of possible knowledge without trigger from the textual level. In other words, an overuse of the inferential capacity, viz. a processing account without considering the actual linguistic trigger, would unwisely lift the processing effort and would as a result cause unwanted low relevance. Thus, an empirical approach to polysemy that pays attention to the co-text proves most relevant and appropriate. ${ }^{17}$

\section{Conclusion}

The present model of prepositional polysemy is a highly contextualizing approach that attempts to bridge the gap between pragmatics and cognitive linguistics by

${ }_{17}$ For a similar stance, see Lu's (2016) discussion of the polysemy of $u p$, where the author also addresses the role of context and makes a similar use of the rule of parsimony. However, the mechanism of perspectivization does not concern that study. 
partitioning context into linguistic context, physical context, and stored knowledge patterns and by regarding perspective taking also as a pragmatic mechanism in deriving interpretations. With an incorporation of contextualization and perspectivization as such and the principle of parsimony as the fundamental criterion for sense establishment, I advance a methodology that distinguishes context-independent meanings as senses from interpretations. In this spirit, an interpretation is nothing more than an inferential consequence of human cognitive capacity emerging from the combination of the proto-scene, vantage point choice, and the various contextual factors. Such argument renders the representation of polysemy highly economical and highlights the role of pragmatics as an information processing system (Sperber, Wilson 1986, 2002; Carston 2002). Despite its advantage of putting rampant polysemy under control, the present model stresses the importance of textual prompts to prevent the inferential capacity from being exaggerated in sacrifice of relevance due to undesired high processing effort. However, future studies are certainly needed to attest the validity of the proposal and to further elaborate on the relation between pragmatics and current cognitive models of language and thought. It is also my hope that this cognitive pragmatic approach to polysemy may contribute not only to establishment of a cognitive linguistic model of polysemy but to delineate the interface of language use and cognition from a more contextually sensitive approach giving due attention to pragmatics.

\section{Acknowledgements}

A working version of this paper appeared in vol. vil of Theory and practice in English studies (mainly Section 3 of the present paper). I thank the student editor, Ms. Veronika Pituková for a few useful copy-editing suggestions. I also thank I-wen Su for valuable suggestions for improving the draft, with the typical disclaimer that applies.

\section{References}

Carston R. 2002. Linguistics meaning, communicated meaning and cognitive pragmatics. Mind and Language 17: 127-48.

Croft W., Cruse A. 2004. Cognitive linguistics. Cambridge, New York.

Cruse A. 1986. Lexical semantics. Cambridge, New York.

Dirven R. 1993. Dividing up physical and mental space into conceptual categories by means of English prepositions. - Zelinsky-Wibbelt C. (ed.). The semantics of prepositions: From mental processing to natural language. Berlin: 73-97.

Evans V., Tyler A. 2004. Spatial experience, lexical structure and motivation: The case of $i n .-$ Radden G., Panther K. (eds.). Studies in linguistic motivation. Berlin: 157-192.

Fauconnier G., Turner M. 2002. The way we think: Conceptual blending and the mind's hidden complexities. New York.

Grady J. 1997. Foundations of meaning: Primary metaphors and primary scenes. [Ph.D. Diss., Linguistics Department, UC California]. 
Grice P. 1978. Further notes on logic and conversation. - Cole P., Morgan J. (eds.). Syntax and semantics. [vol. 9: Pragmatics]. New York: 113-128.

Hawkins B. 1988. The category MEDIUM. - Rudzka-Ostyn B. (ed.). Topics in cognitive linguistics. Amsterdam: 231-270.

Herskovits A. 1986. Language and spatial cognition: An interdisciplinary study of the prepositions in English. Cambridge.

Herskovits A. 1988. Spatial expressions and the plasticity of meaning. - Rudzka-Ostyn B. (ed.). Topics in cognitive linguistics. Amsterdam: 271-298.

Hottenroth P.-M. 1993. Prepositions and object concepts: A contribution to cognitive semantics. - Zelinsky-Wibbelt C. (ed.). The semantics of prepositions: From mental processing to natural language. Berlin: 179-219.

Johnson M. 1987. The body in the mind. Chicago.

Lakoff G. 1987. Women, fire, and dangerous things: What categories reveal about the mind. Chicago.

Lakoff G., Johnson M. 1980. Metaphors we live by. Chicago.

Lakoff G., Johnson M. 1999. Philosphy in the flesh: The embodied mind and its challenge to Western thought. New York.

Langacker R. 1987. Foundations of Cognitive Grammar. [vol. 1]. Stanford.

Langacker R. 1991. Concept, image, and symbol: The cognitive basis of grammar. Berlin.

Lindstromberg S. 1997. English prepositions explained. Amsterdam.

Lu W. 2008. From textual prompts to cognitive models: A context-oriented perspective on metaphor interpretation in Taiwanese presidential speeches. - Language and Linguistics 9.2: 341-358.

Lu W. 2016. Polysemy and the semantic-pragmatic interface: The case of up in a contextbased model. - Intercultural Pragmatics 13.4: 563-589.

Miller G., Johnson-Laird Ph. 1976. Language and perception. Cambridge.

Pustejovsky J. 1991. The generative lexicon. - Computational Linguistics 17.4: 409-441.

Reddy M. 1979. The conduit metaphor. - Ortony A. (ed.). Metaphor and thought. Cambridge: 284-310.

Rudzka-Ostyn B. 2003. Word power: Phrasal verbs and compounds. New York.

Ruhl Ch. 1989. On monosemy: A study in linguistic semantics. Albany.

Sandra D. 1998. What linguists can and can't tell about the mind: A reply to Croft. - Cognitive Linguistics 9.4: 361-378.

Sperber D., Wilson D. 1986. Relevance: Communication and cognition. Oxford.

Sperber D., Wilson D. 2002. Pragmatics, modularity and mindreading. - Mind \& Language 17: 3-23.

Taylor J. 2003. Polysemy's paradoxes. - Language Sciences 25: 637-655.

Tyler A., Evans V. 2003. The semantics of English prepositions: Spatial scenes, embodied meaning and cognition. Cambridge, New York.

Vandeloise C. 1991. Spatial prepositions: A case study in French. Chicago.

Vandeloise C. 1994. Methodology and analyses of the preposition in. - Cognitive Linguistics 5.2: $157-184$.

Wilson D. 2003. Relevance theory and lexical pragmatics. - Italian Journal of Linguistics / Rivista di Linguistica 15.2: 273-291. 